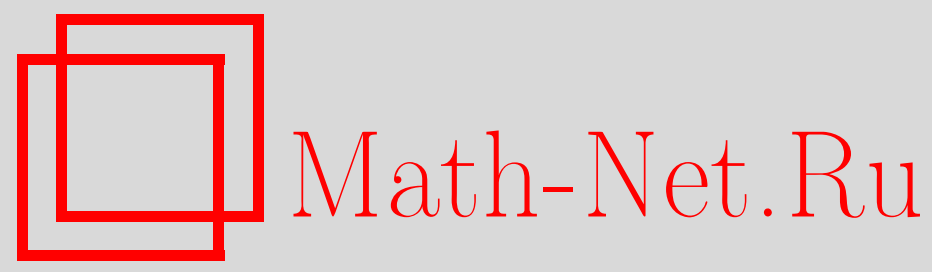

А. В. Полбин, Н. Д. Фокин, Эконометрическое моделирование сбалансированной структурной компоненты основных российских макроэкономических показателей, Матем. моделирование, 2020, том 32, номер 7, 98-112

DOI: https://doi.org/10.20948/mm-2020-07-06

Использование Общероссийского математического портала Math-Net.Ru подразумевает, что вы прочитали и согласны с пользовательским соглашением http://www.mathnet.ru/rus/agreement

Параметры загрузки:

IP : 54.89 .56 .158

26 апреля 2023 г., $17: 36: 56$

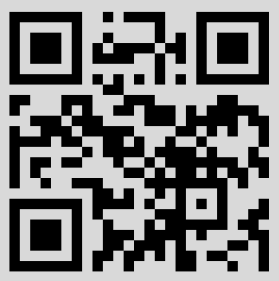




\title{
ЭКОНОМЕТРИЧЕСКОЕ МОДЕЛИРОВАНИЕ СБАЛАНСИРОВАННОЙ СТРУКТУРНОЙ КОМПОНЕНТЫ ОСНОВНЫХ РОССИЙСКИХ МАКРОЭКОНОМИЧЕСКИХ ПОКАЗАТЕЛЕЙ
}

\author{
(C) 2020 2. $\quad$ А.В. Полбин ${ }^{1,2}$, Н.Д. Фокин ${ }^{1}$
}

${ }^{1}$ Российская академия народного хозяйства и государственной службы при Президенте Российской Федерации

${ }^{2}$ Институт экономической политики имени Е.Т. Гайдара apolbin@gmail.com, fokinikita@gmail.com

DOI: $10.20948 / \mathrm{mm}-2020-07-06$

B работе предложена модель векторной авторегрессии (VAR) с дополнительной задачей регуляризации по типу задачи фильтра Ходрика-Прескотта для моделирования единого, т.е. сбалансированного долгосрочного темпа роста структурной компоненты основных макроэкономических показателей российской экономики. В модели участвуют: реальный ВВП без государственных расходов, реальное потребление домашних хозяйств, реальные инвестиции в основной капитал, реальный экспорт, реальный импорт и реальный эффективный обменный курс рубля. Также в модель экзогенно включены цены на нефть. Предполагается, что ВВП без госрасходов и его составляющие имеют единый потенциальный темп роста, а отличия в фактически достигнутом увеличении макроэкономических показателей объясняются разными долгосрочными мультипликаторами по ценам на нефть, а также случайными шоками. На основе предложенной модели мы рассчитываем вклады цен на нефть и структурной компоненты в динамику ВВП без госрасходов и его составляющих.

Ключевые слова: потенциальный темп роста, российская экономика, цены на нефть.

\section{ECONOMETRIC MODELING OF THE BALANCED POTENTIAL GROWTH RATE OF THE MAIN RUSSIAN MACROECONOMICS VARIABLES}

\author{
A.V. Polbin ${ }^{1,2}$, N.D. Fokin ${ }^{1}$ \\ ${ }^{1}$ Russian Academy of National Economy and Public Administration \\ ${ }^{2}$ Gaidar Institute for Economic Policy
}

The paper provides vector autoregression model with an additional regularization problem similar to the Hodrick-Prescott filter problem for modeling a single, i.e. a balanced growth rate of the structural component of the main macroeconomic indicators of the Russian economy. The model includes real GDP without government consumption, real 
household consumption, real fixed capital investment, real export, real import and the real effective ruble exchange rate. The oil prices are exogenously included in the model. It is assumed that GDP without government consumption and its components have balanced potential growth rate. The actual discrepancies in time series are explained by the different long-term oil prices multipliers, and by the stochastic shocks. Based on the proposed model we calculate the impacts of the oil prices shocks and the structural component on GDP without government consumption and its components.

Key words: potential growth rate, Russian economy, oil prices.

\section{1. Введение}

При выработке мер денежно-кредитной политики центральные банки и другие институты экономической политики в современных условиях опираются на формальные экономико-математические модели и на широкий набор индикаторов экономической активности. Примерами наиболее важных таких индикаторов являются циклическая компонента выпуска, потенциальный (долгосрочный или структурный) уровень выпуска и темп долгосрочного роста. Данные компоненты являются ненаблюдаемыми величинами и их необходимо оценивать с помощью статистических методов. Однако простое применение популярных фильтров, не учитывая специфику анализируемого макроэкономического показателя, может порождать ложные циклические колебания и приводить к неверным выводам для экономической политики. Это обосновывает актуальность разработки методов выделения упомянутых выше компонент, которые учитывают статистические характеристики анализируемых временных рядов. Другой важный аспект заключается в том, что ВВП является не единственным, а даже, возможно, не наилучшим показателем, характеризующим стадию делового цикла. Например, во время кризисов в наибольшей степени падают инвестиции, а не потребление или ВВП. Таким образом, актуальным является использование как можно большего объема информации, большего количества временных рядов статистики национальных счетов для выделения трендовой и/или циклической составляющих.

Для выделения трендовой составляющей существует большой набор эконометрических методов, которые условно можно разделить на структурные и неструктурные. Под неструктурными методами подразумеваются подходы, не использующие содержательных предпосылок экономической теории об анализируемом временном ряде, а также обычно использующие всего один временной ряд. К неструктурным методам относятся различные процедуры сглаживания и фильтры, например, скользящее среднее, фильтр 
Ходрика-Прескотта (НР фильтр) [1], полосовой фильтр Бакстера-Кинга [2], декомпозиция Бевериджа-Нельсона [3], фильтр Калмана.

Под структурными методами подразумеваются процедуры, использующие предпосылки экономической теории, а также дополнительные временные ряды. Например, данные по инфляции и инфляционным ожиданиям или безработице. Используя такие временные ряды, можно выделять потенциальный выпуск с помощью теоретических экономических концепций, такие как кривая Филлипса или закон Оукена [4]. Отметим, что для выделения структурной компоненты также могут применяться классические эконометрические модели на основе структурных уравнений, где ВВП является производственной функцией от капитала, труда и совокупной факторной производительности [5]. Многомерный фильтр Ходрика-Прескота и многомерный фильтр Калмана также относят к структурным методам. Многомерный фильтр Ходрика-Прескота использовался, например, в [6]. В последней работе, как и в работах $[7,8]$, к ряду российского ВВП применялся также многомерный фильтр Калмана.

В данной работе мы предлагаем модификацию классического фильтра Ходрика-Прескотта для выделения единого долгосрочного темпа роста основных реальных российских макроэкономических показателей, таких как: ВВП без госрасходов (пояснение для выбора ВВП без госрасходов будет дано ниже), потребление домохозяйств, инвестиции в основной капитал, экспорт и импорт. В качестве показателя конкурентоспособности российской экономики в модели участвует реальный эффективный обменный курс рубля, а также экзогенные реальные цены на нефть, скорректированные на долларовый индекс потребительских цен. Таким образом, мы ставим задачу выделения единого динамического долгосрочного темпа роста для ВВП без госрасходов и его составляющих, участвующих в моделях, при этом контролируя на цены на нефть, кросс-корреляционные взаимосвязи и реальный обменный курс рубля.

Предпосылка о сбалансированном росте является вполне классической в динамических моделях экономического роста. Она означает, что основные переменные модели, например, выпуск, потребление и инвестиции растут единым темпом. Однако в эконометрических работах она учитывается довольно редко, хотя для ее учета в эконометрических моделях достаточно наложить некоторое число нелинейных ограничений на параметры модели. К примеру, ее реализация достаточно популярна в байесовских VAR [9]. Учитывая, что в векторных авторегрессиях долгосрочные прогнозы сходятся к безусловному среднему переменных модели, корректная оценка сред- 
него является критически важной задачей. В [10] авторы показали, что основной причиной плохих долгосрочных прогнозов как раз является плохая оценка среднего. В связи с этим, основная задача модели, предложенной в данной работе, - выделение некоторого динамического сбалансированного среднего темпа роста ВВП без госрасходов и его составляющих, очищенного от влияния цен на нефть и кросс-корреляционных взаимосвязей.

Включение в модель вышеупомянутых переменных следует из достаточно простых спецификаций теоретических моделей общего равновесия, таких как DSGE. В [11] предложена модель закрытой экономики, в которой «частный» выпуск (ВВП за исключением государственных расходов), потребление домашних хозяйств и инвестиции в основной капитал имеют единый долгосрочный стохастический тренд, наличие которого обусловлено стохастическим трендом во временном ряде совокупной факторной производительности. В [12] предлагается расширение модели из работы [11] для открытой экономики, в которой при неизменных условиях торговли такие переменные, как ВВП без госрасходов, валовое накопление основного капитала, потребление домохозяйств, экспорт и импорт разделяют единый стохастический тренд, реальный обменный курс же не имеет трендовой динамики. Изменяющиеся же во времени нестационарным образом условия торговли в предложенной теоретической модели оказывают перманентное влияние на все отечественные макроэкономические показатели.

Государственные расходы исключаются из ряда выпуска по причине того, что они не следуют общему тренду производительности и процесс их формирования существенно отличается от образования вышеупомянутых переменных. Так, например, в России реальные государственные расходы практически не меняются во времени, а доля номинальных госрасходов в номинальном ВВП предельно стабильна и колеблется в среднем около уровня в 17\% за период 1999-2019 гг. Это обусловлено особенностями методологии построения госрасходов в постоянных ценах: изменение во времени данного показателя во многом измеряется изменением занятости в госсекторе. При необходимости построения прогноза для ВВП с помощью предлагаемой модели возможно построить прогноз для ВВП без госрасходов на основе настоящей модели, прогноз госрасходов на основе некоторой внешней модели и далее провести их агрегирование. Полученные оценки долгосрочных темпов роста могут найти практическую ценность при калибровке параметров DSGE модели для российской экономики, отвечающих за долгосрочные темпы роста, что необходимо для построения более точных прогнозов. 
Работа построена следующим образом: во втором разделе мы описываем методологию фильтра Ходрика-Прескотта, в третьем разделе - постановку VAR модели с HP фильтром, в четвертом разделе мы описываем используемые в работе данные, сравниваем результаты по оцененной модели с классическим фильтром Ходрика-Прескотта, а также рассчитываем исторические вклады долгосрочного темпа роста и цен на нефть в динамику переменных модели.

\section{2. Фильтр Ходрика-Прескотта}

В оригинальной работе [1] авторы исследовали временной ряд реального валового национального продукта США за период 1950-1979 гг. Авторами ставилась задача декомпозиции очищенного от сезонного фактора ряда ВНП $y_{t}$ на структурную $\mu_{t}$ и циклическую $c_{t}$ компоненты. При этом фактический ряд $y_{t}$ есть в точности сумма $\mu_{t}$ и $c_{t}$. В оптимизационной задаче данного фильтра минимизируется квадрат циклической компоненты, а также функционал штрафуется за величину квадрата второй разности структурной компоненты, то есть в задаче вводится штраф за негладкость долгосрочных темпов роста. Данную задачу можно записать в следующем виде:

$$
\sum_{t=1}^{T}\left(y_{t}-\mu_{t}\right)^{2}+\lambda \sum_{t=3}^{T}\left[\left(\mu_{t}-\mu_{t-1}\right)-\left(\mu_{t-1}-\mu_{t-2}\right)\right]^{2} \rightarrow \min _{\left\{\mu_{t}\right\}_{t=1}^{T}},
$$

где $T$ - число наблюдений, а $\lambda$ - гиперпараметр, принимающий положительные значения и отвечающий за гладкость структурной компоненты. Значение данного параметра влияет на то, как часто фильтр диагностирует периоды, когда уровень фактического ряда ниже структурного, и перегревы экономики (когда уровень фактического ряда выше структурного). В случае если $\lambda=0$, структурная компонента будет в точности совпадать с анализируемым рядом. В случае если $\lambda \rightarrow \infty$, структурная компонента будет стремиться к линейному тренду. В оригинальной работе параметр $\lambda$ для американской экономики устанавливался вручную. Для годовых данных рекомендуется использовать значение 100, для квартальных данных - значение 1600 , а для месячных - значение 14400. Однако данная рекомендация носит условный характер, так как в других странах экономические циклы могут возникать с иной периодичностью.

Неопределенность в выборе значения параметра $\lambda$ является одним из недостатков фильтра Ходрика-Прескотта. Однако данная проблема может быть решена путем выбора значения гиперпараметра на основе скользящей кросс-валидации. 
Еще одним недостатком данного фильтра является проблема крайних точек. Она заключается в том, что оценки структурной компоненты на первых и последних точках чувствительны к добавлению новых наблюдений, т.е. если добавить в выборку несколько новых точек в начале и в конце временного ряда, переоцененный на более полной выборке уровень структурной компоненты может сильно отличаться от оценки на меньшей выборке.

\section{3. Постановка VAR модели}

Классическая задача OLS оценки VAR модели состоит в минимизации суммы квадратов ошибок каждого уравнения по отдельности. Запишем VAR модель из $k$ переменных с константами, $p$ эндогенными лагами и $q$ лагами $s$ экзогенных переменных:

$$
\begin{aligned}
& Y_{t}=C+\sum_{j=1}^{p} A_{j} Y_{t-j}+\sum_{j=0}^{q} B_{j} X_{t-j}+\varepsilon_{t}, \quad t=1, \ldots, T, \\
& Y_{t}=\left(\begin{array}{c}
y_{1, t} \\
\vdots \\
y_{k, t}
\end{array}\right), \quad C=\left(\begin{array}{c}
c_{1} \\
\vdots \\
c_{k}
\end{array}\right), \quad X_{t}=\left(\begin{array}{c}
x_{1, t} \\
\vdots \\
x_{s, t}
\end{array}\right), \quad \varepsilon_{t}=\left(\begin{array}{c}
\varepsilon_{1, t} \\
\vdots \\
\varepsilon_{k, t}
\end{array}\right),
\end{aligned}
$$

где $C$ - вектор констант, $A_{j}$ - матрица параметров при эндогенных переменных на лаге $j$ :

$$
A_{j}=\left(\begin{array}{ccc}
a_{11, j} & \ldots & a_{k 1, j} \\
\vdots & \ddots & \vdots \\
a_{k 1, j} & \cdots & a_{k k, j}
\end{array}\right),
$$

$B_{j}$ - матрица параметров при экзогенных переменных на лаге $j$ :

$$
B_{j}=\left(\begin{array}{ccc}
b_{11, j} & \ldots & b_{1 s, j} \\
\vdots & \ddots & \vdots \\
b_{k 1, j} & \cdots & b_{k s, j}
\end{array}\right) .
$$

При условии, что все переменные вектора $Y_{t}$ стационарны, т.е. имеют некоторое математическое ожидание и дисперсию, которые не зависят от времени, а также $\forall i \in\{1, \ldots, k\} \operatorname{Cov}\left(y_{i, t}, y_{i, t-\tau}\right)$ не зависит от времени, а зависит только от $\tau$, безусловным математическим ожиданием вектора $y_{t}$ будет величина $\mu$ :

$$
\mu=E\left(y_{t}\right)=\left(I-\sum_{j=1}^{p} A_{j}\right)^{-1} C+\left(I-\sum_{j=1}^{p} A_{j}\right)^{-1} \sum_{j=0}^{q} B_{j} E\left(X_{t-j}\right),
$$

где $I$ - единичная матрица. 
В такой модели, если ряд стационарный, например, темп роста ВВП, безусловное математическое ожидание является некоторой оценкой потенциального темпа роста, так как безусловный долгосрочный прогноз по данной модели равен безусловному математическому ожиданию. То есть при отсутствии шоков переменные модели растут некоторым постоянным потенциальным темпом роста в долгосрочной перспективе. Учет предпосылки о сбалансированном росте можно реализовать наложением ряда нелинейных ограничений на параметры модели.

Мы предлагаем модификацию VAR модели, в которой параметр, отвечающий за некоторый средний уровень временного ряда, будет меняться во времени. При этом мы ставим задачу выделения сбалансированного, то есть единого потенциального темпа роста ВВП без госрасходов, потребления, инвестиций, экспорта и импорта, очищенного от цен на нефть. К задаче метода наименьших квадратов добавляется задача регуляризации, аналогичная фильтру Ходрика-Прескотта. Такую VAR модель можно записать в виде

$$
Y_{t}-\mu_{t}=\sum_{j=1}^{p} A_{j}\left(Y_{t-j}-\mu_{t-j}\right)+\sum_{j=0}^{q} B_{j} X_{t-j}+\varepsilon_{t},
$$

где $Y_{t}, A_{j}, B_{j}, X_{t}, \varepsilon_{t}$ аналогичны обычной VAR модели (2). Задача оптимизации для данной модели:

$$
\sum_{j=1}^{k} \sum_{t=1}^{T} \varepsilon_{j, t}^{2}+\lambda \sum_{t=2}^{T}\left[\left(\mu_{t}-\mu_{t-1}\right)\right]^{2} \rightarrow \min _{\left\{\mu_{t}\right\}_{t=1}^{T}} .
$$

Так как мы рассматриваем модель в разностях и оцениваем потенциальный темп роста, а фильтр Ходрика-Прескотта - потенциальный уровень, в данной модели $\mu_{t}$ является потенциальным темпом роста, а не уровнем, и следовательно, мы штрафуем квадрат его первой разности, а не второй.

Модель (8) оценивается с помощью функции fmincon в Matlab c использованием метода концентрации. На первом шаге задается некоторое значение гиперпараметра $\lambda$. На втором шаге производится численная минимизация по вектору $\mu_{t}$, в рамках которой при каждом значении $\mu_{t}$ оценки коэффициентов матриц $A_{j}$ и $B_{j}$ рассчитываются как OLS оценки регрессии центрированных временных рядов $Y_{t}$ на $\mu_{t}$, лаги данных центрированных временных рядов $X_{t}$ и лаги $X_{t}$ в модели без свободного члена.

\section{4. Данные и результаты оценивания}

Данные по реальному ВВП и его компонентам собраны с сайта Росстата, данные по реальному эффективному обменному курсу рубля - с сайта Международного валютного фонда, данные по цене на нефть и долларово- 
му индексу потребительских цен - с сайта Федеральной резервной системы США. Все ряды в квартальном выражении с первого квартала 1999 г. по третий квартал 2019 г. Все переменные включены в разностях логарифмов и очищены от сезонности процедурой ARIMA X-12 (кроме реального курса и номинальной цены на нефть). У ВВП без государственных расходов, потребления домашних хозяйств, инвестиций в основной капитал, экспорта и импорта предполагается единый потенциальный темп роста $\mu_{t}$, а у реального обменного курса предполагается нулевой потенциальный темп роста, то есть данный показатель в долгосрочной перспективе не меняется при неизменности переменных модели.

Мы используем данные по ВВП по использованию в четырех разных представлениях: за период 1995-2008 гг. в ценах 2003 г., за период 20022011 гг. в ценах 2008 г., за период 2011-2016 гг. в ценах 2011 г. и за период 2014-2019 гг. в ценах 2016 г. Для любой пары “соседних” уровней цен (2003 и 2008, 2008 и 2011, 2011 и 2016) значение ряда в новых ценах получается на основе расчета годового темпа роста ряда в старых ценах, умноженного на прошлогоднее значение ряда в новых ценах. Аналогичный подход сцепки временных рядов использовался, например, в [13]. Данная процедура позволяет получить ряды реального ВВП и его компонент за максимальный широкий период времени, который публикует Росстат.

Удаление ряда реальных госрасходов из ряда реального ВВП простым вычитанием некорректно, так как реальные показатели Росстат рассчитывает с помощью индекса Ласпейреса. В данной работе удаление госрасходов из ВВП произведено по методологии из работы [14].

На основе одношаговой скользящей кросс-валидации на периоде (2014q42019q3) мы выбрали оптимальное значение $\lambda=370$, которое минимизирует среднеквадратичную ошибку прогноза модели на данном периоде. В качестве общей ошибки прогноза модели (т.к. у переменных модели разные дисперсии) используется среднеквадратичная ошибка прогноза по всем переменным, нормированным на их стандартные отклонения. С полученным значением гиперпараметра модель оценивается на полной выборке (1999q12019q3). В модели участвует всего один лаг эндогенных переменных, а также разность логарифма цен на нефть и первый лаг разности логарифма цен на нефть, т.е. на 83, а строго говоря, на 81 наблюдении (две точки пропадают за счет взятия первых разностей и первых лагов) необходимо оценить 8 параметров в каждом уравнении помимо вектора $\mu_{t}$. Также мы сопоставляем результаты VAR-HP модели с классическим фильтром Ходрика-Прескотта. На рис.1 изображены фактические темпы роста, темпы роста структурной ком- 
поненты по VAR-HP фильтру и HP фильтру. Фильтр Ходрика-Прескотта реализуется в логарифмах уровней рассматриваемых рядов, а затем от уровня структурной компоненты берется первая разность, тем самым получается темп роста структурной компоненты.
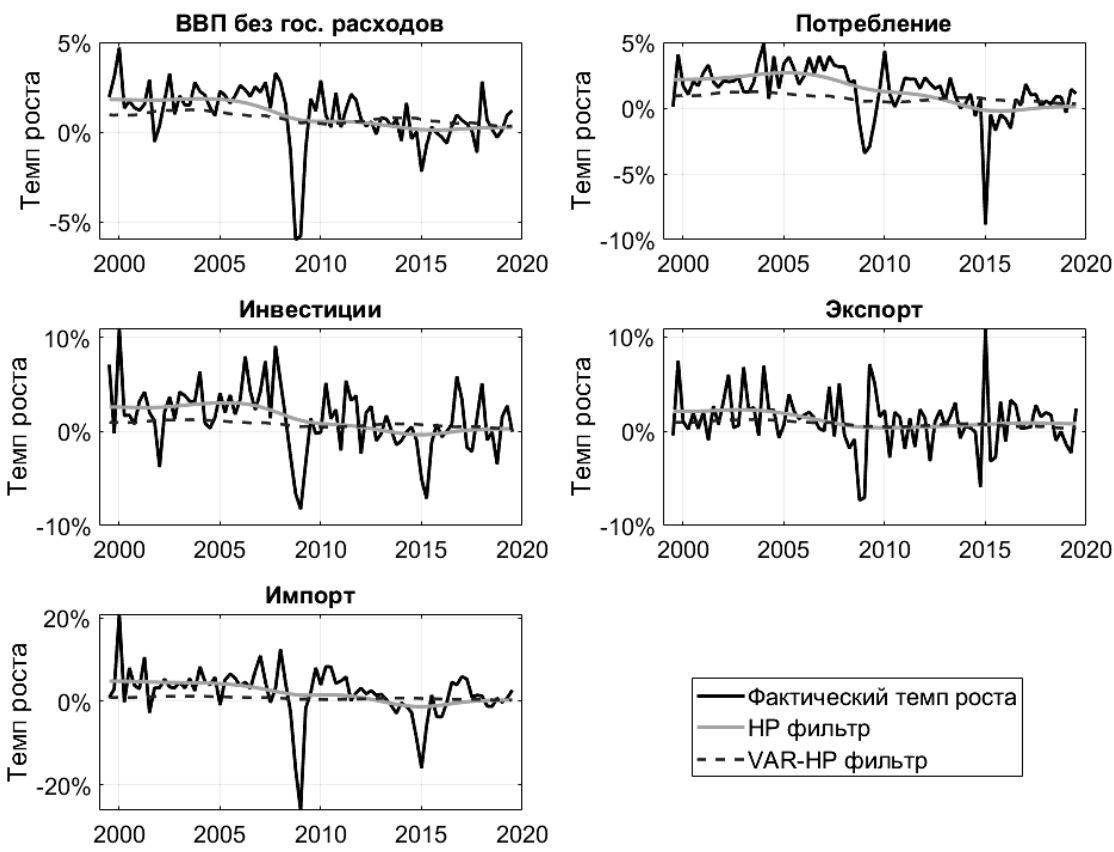

Рис.1. Сопоставление фактических и потенциальных темпов роста на основе HP и VAR-HP фильтров.

Результаты для всех рядов достаточно похожи, в том смысле, что в период 1999-2011 гг. НР фильтр оценивает темп роста структурной компоненты значительно выше, чем VAR-HP фильтр, однако после 2011 г. соотношение оценок меняется - потенциальный темп роста на основе VAR-HP фильтра оказывается значительно выше потенциального темпа роста на основе VAR-HP. При этом на основе VAR-HP модели в третьем квартале 2019г. потенциальный темп роста российской экономики оценивается в $1.36 \%$ в год, а в начале рассматриваемого периода он составлял $4.1 \%$ в год.

Учитывая, что фильтр Ходрика-Прескотта выделяет динамический средний уровень, не учитывая изменений цен на нефть, данный результат можно интерпретировать следующим образом: в целом быстрорастущие цены на нефть в 2000-2008 гг. вносили большой вклад в динамику рассматриваемых показателей. Когда они резко упали во время кризиса 2008-2009 гг., потенциальный темп роста на основе НР фильтра также несколько замедлился. Однако существует и еще один фактор, за счет которого могло про- 
изойти замедление во время кризиса 2008-2009 гг. Например, в [15] авторы идентифицируют сдвиг именно в темпе роста структурной компоненты российского выпуска в конце 2007г. Таким образом, имело место одновременное замедление потенциального темпа роста российской экономики, а также падение цен на нефть. Преимущество VAR-HP фильтра состоит в том, что данная модель может разделить оба этих эффекта, когда классический НР фильтр считает, что сокращалась только структурная компонента, так как цены на нефть в нем не учитываются.

Кроме того, на рисунке видно, что, например, в случае самого волатильного показателя - импорта - потенциальный темп роста на основе НР фильтра в начале рассматриваемого периода составляет почти 5\% в квартал, когда сбалансированный потенциальный темп роста на основе VAR-HP в аналогичный момент времени составляет всего 1\% в квартал. Такая оценка может показаться очень низкой для показателя импорта, который может измениться на $10 \%$ или даже $20 \%$ за квартал. Однако если мы рассчитаем долгосрочные мультипликаторы рассматриваемых показателей по ценам на нефть, мы получим результаты, которые в целом объясняют данную ситуацию. В табл.1 приведены рассчитанные долгосрочные изменения переменных модели в ответ на 10\% перманентный шок реальной цены на нефть. Под перманентным шоком подразумевается такое изменение цен на нефть, в результате которого цена на нефть закрепляется на некотором новом уровне, который равен $110 \%$ от старого, например, поднимается с $\$ 50$ за баррель и остается на уровне $\$ 55$ за баррель.

Таблица 1. Долгосрочные реакции переменных модели на 10\% перманентный шок цен на нефть и 68\% асимптотические нормальные доверительные интервалы

\begin{tabular}{|c|c|c|c|c|c|c|}
\hline & $\begin{array}{c}\text { ВВП без } \\
\text { госрасходов }\end{array}$ & $\begin{array}{c}\text { Потребле- } \\
\text { ние }\end{array}$ & $\begin{array}{c}\text { Инвести- } \\
\text { ции }\end{array}$ & Экспорт & Импорт & $\begin{array}{c}\text { Реальный } \\
\text { курс }\end{array}$ \\
\hline $\begin{array}{c}\text { оцен- } \\
\text { ка }\end{array}$ & 1.19 & 1.34 & 2.35 & 0.87 & 4.59 & 1.83 \\
{$[0.98 ; 1.40]$} & {$[1.08 ; 1.60]$} & {$[1.96 ; 2.73]$} & {$[0.66 ; 1.08]$} & {$[3.93 ; 5.30]$} & {$[1.31 ; 2.35]$} \\
\hline
\end{tabular}

Из таблицы следует, что при 10\% шоке цен на нефть ВВП без госрасходов в долгосрочной перспективе увеличивается на $1.2 \%$, а, например, импорт, о котором мы писали выше, почти на 5\%. Таким образом, изменяющаяся цена на нефть вносила разные вклады в анализируемые ряды. Изменение потребления при изменении цены на нефть на $10 \%$ составляет $1.34 \%$ и близко к изменению ВВП без госрасходов, изменение инвестиций составляет $2.35 \%$, изменение экспорта составляет $0.87 \%$. Для того чтобы проанализировать влияние разделенных эффектов цен на нефть и изменения структурной компоненты, обратимся к табл.2, в которой рассчитаны кумулятивные вклады двух данных факторов за различные периоды времени. 
Таблица 2. Кумулятивные вклады потенциального темпа роста и цен на нефть за разные временные промежутки.

\begin{tabular}{|c|c|c|c|c|c|}
\hline & $\begin{array}{c}\text { ВВП без } \\
\text { госрасходов }\end{array}$ & Потребление & Инвестиции & Экспорт & Импорт \\
\hline $\begin{array}{l}\text { Рост за период } \\
2000 \mathrm{q} 1-2008 \mathrm{q} 2\end{array}$ & $66 \%$ & $86 \%$ & $111 \%$ & $61 \%$ & $171 \%$ \\
\hline $\begin{array}{c}\text { За счет цен на } \\
\text { нефть }\end{array}$ & $17 \%$ & $19 \%$ & $33 \%$ & $12 \%$ & $65 \%$ \\
\hline $\begin{array}{l}\text { За счет структур- } \\
\text { ной компоненты }\end{array}$ & \multicolumn{5}{|c|}{$35 \%$} \\
\hline $\begin{array}{l}\text { Рост за период } \\
2000 q 1-2014 q 4\end{array}$ & $72 \%$ & $109 \%$ & $110 \%$ & $63 \%$ & $167 \%$ \\
\hline $\begin{array}{c}\text { За счет цен на } \\
\text { нефть }\end{array}$ & $15 \%$ & $18 \%$ & $31 \%$ & $7 \%$ & $57 \%$ \\
\hline $\begin{array}{l}\text { За счет структур- } \\
\text { ной компоненты }\end{array}$ & \multicolumn{5}{|c|}{$52 \%$} \\
\hline $\begin{array}{l}\text { Рост за период } \\
2000 q 1-2019 q 3\end{array}$ & $75 \%$ & $104 \%$ & $109 \%$ & $83 \%$ & $164 \%$ \\
\hline $\begin{array}{c}\text { За счет цен на } \\
\text { нефть }\end{array}$ & $10 \%$ & $12 \%$ & $20 \%$ & $5 \%$ & $38 \%$ \\
\hline $\begin{array}{l}\text { За счет структур- } \\
\text { ной компоненты }\end{array}$ & \multicolumn{5}{|c|}{$61 \%$} \\
\hline
\end{tabular}

Мы рассматриваем три периода - первый с 2000 г. до момента рецессии во время мирового финансового кризиса в 2008-2009 гг. (третий квартал 2008 г.). Второй период - с 2000 г. до момента рецессии 2015 г. - четвертый квартал 2014 г., в первом квартале 2015 г. уже произошло сокращение сезонно очищенного ВВП без госрасходов на $2.2 \%$. И третий период - весь рассмотренный нами временной промежуток, начиная с первого квартала 2000г. На основе полученных оценок за первый период ВВП без госрасходов вырос на 66\%, из которых 17\% были обусловлены ростом цен на нефть, а 35\% - ростом структурной компоненты. Во втором периоде, учитывая, что вклад цены на нефть был сильно отрицательным в период мирового финансового кризиса, цены на нефть внесли всего $15 \%$ в рост ВВП без госрасходов, когда сам валовый выпуск вырос на $72 \%$, в том числе за счет структурной компоненты на 52\%. Если говорить обо всем рассмотренном периоде, ВВП без госрасходов вырос на 75\%, из которых 10\% обусловлены ценами на нефть, а $61 \%$ - структурной компонентой. Т.е. доминирующую роль в росте выпуска играет структурная компонента, темп роста которой на данный момент находится на рекордно низком историческом уровне, согласно оценкам модели.

Если взять совокупный рост за весь период за 100, то цены на нефть кумулятивно внесли почти $13 \%$ в рост ВВП без госрасходов, чуть меньше $12 \%$ - в рост потребления домохозяйств, около одной пятой - в рост инве- 
стиций в основной капитал и импорта и почти 6\% - в рост реального экспорта. Структурная компонента объясняет $81 \%$ роста ВВП без госрасходов, около $59 \%$ роста потребления, $56 \%$ роста инвестиций, $37 \%$ роста импорта и $73 \%$ роста реального экспорта.

Заметим, что уменьшение кумулятивного вклада цен на нефть нельзя воспринимать как свидетельство в пользу того, что российская экономика стала меньше зависеть от цен на нефть. Цены на нефть действительно объясняют меньше динамики ВВП без госрасходов за период 2000-2019, чем за период 2000-2008, что связано с тем, что в моменты рецессий цены на нефть вносят отрицательный вклад, причем, чем больше долгосрочный мультипликатор, тем больше по модулю отрицательный вклад. Этим фактом можно объяснить, почему во время кризисов в наибольшей степени падают, например, инвестиции и импорт.

При этом замедление как фактических, так и структурных темпов роста российской экономики представляются весьма существенными. Если мы исключим из рассмотрения периоды спада в 2008-2009 гг. и в 2015 г., а именно периоды 2008q3-2009q2 и 2015q1-2015q2 и рассчитаем, на сколько за промежутки из табл.1 изменился ВВП без госрасходов, мы получим, что за первый промежуток длиной в 8.5 лет изменение составило все также $66 \%$ (7.8\% в год в среднем), а за последующие 5.5 лет (2009q3-2014q4) ВВП без госрасходов вырос сверх уровня 2009q3 всего на 18\% (84\% за весь период $2000 q 1-2014 q 4$ без кризисных точек). Это означает, что ВВП без госрасходов в среднем рос на $3.2 \%$ в год. При этом цены на нефть в период 2009q3$2014 q 4$ оставались предельно высокими - \$97 за баррель в среднем за период. За четырехлетний период 2015q3-2019q3, сразу после рецессии первых двух кварталов 2015 г., ВВП без госрасходов прирос к уровню 2014q4 всего на 6\%, что меньше, чем средний рост за один год в 2000-2008 гг. Остальные показатели, кроме экспорта, а именно, потребление, инвестиции и импорт, вовсе находятся на уровне ниже, чем 4 года назад. Существенный рост экспорта за данный период, скорее всего, вызван двукратной девальвацией рубля в 2015 г.

Таким образом, представляются очевидными структурные проблемы в российской экономике, которые появились еще 10 лет назад, а усугубились в окрестности кризисного 2015 года и политика органов экономических властей должна быть направлена непосредственно на увеличение темпов структурного роста российской экономики. Дополнительно на рис.2-6 наглядно представлены вклады цен на нефть и структурной компоненты в фактические темпы роста рассматриваемых переменных. 


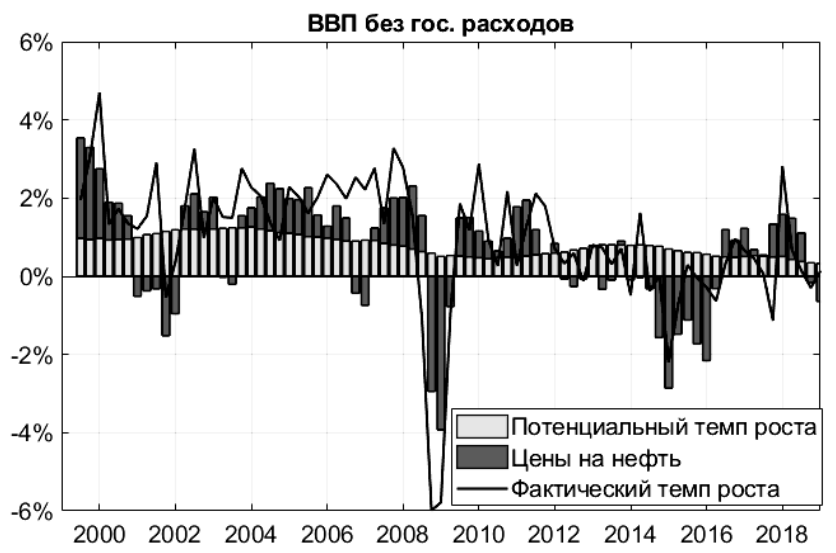

Рис.2. Вклад потенциального темпа роста и цен на нефть в динамику ВВП без госрасходов.

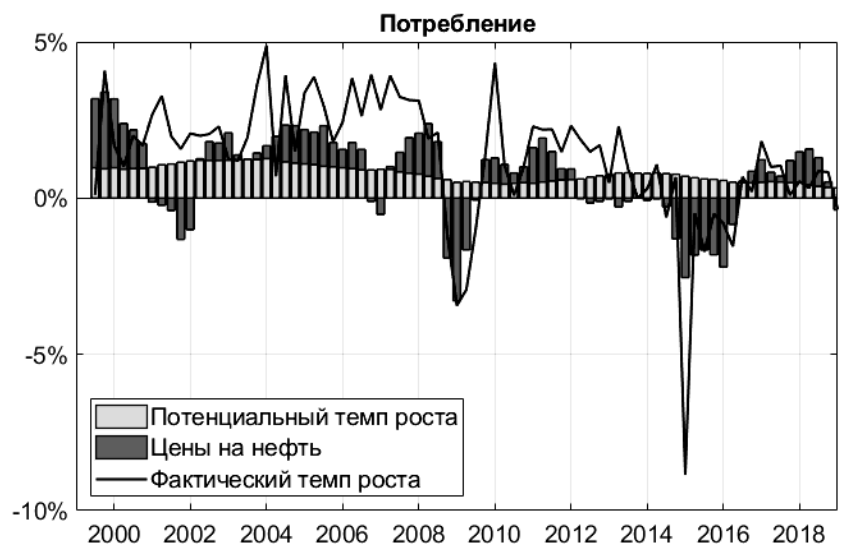

Рис.3. Вклад потенциального темпа роста и цен на нефть в динамику потребления.

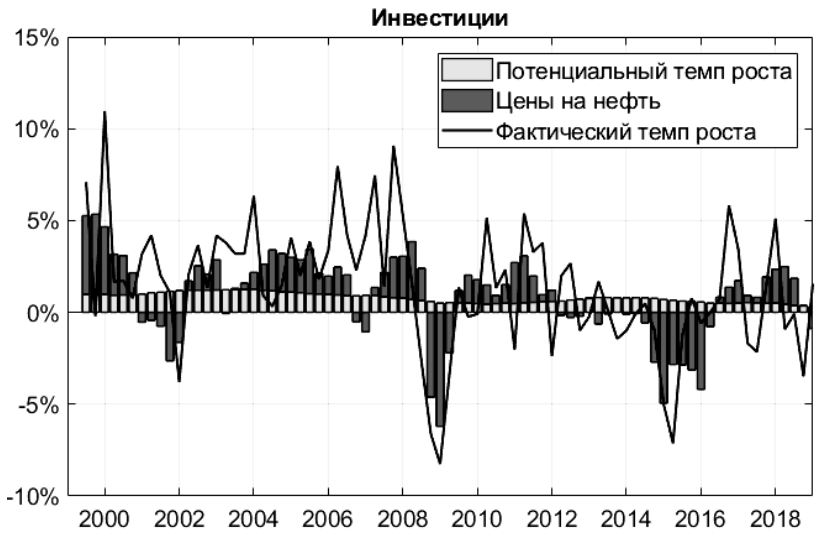

Рис.4. Вклад потенциального темпа роста и цен на нефть в динамику инвестиций. 


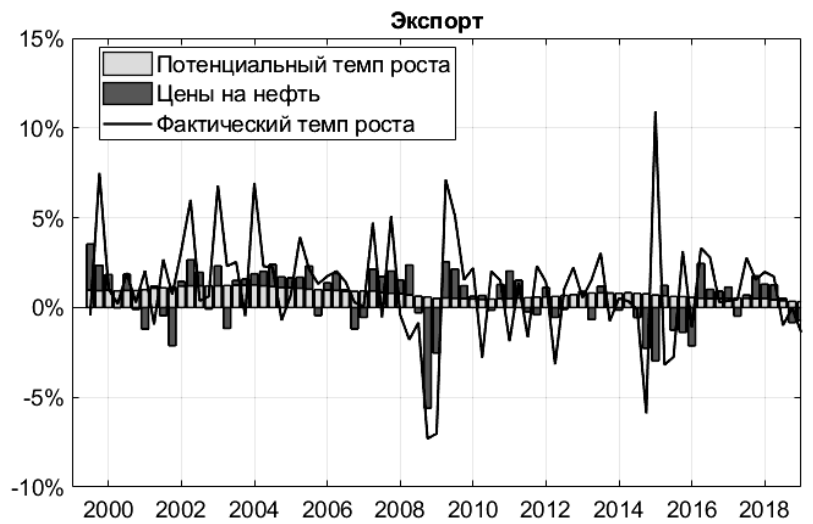

Рис.5. Вклад потенциального темпа роста и цен на нефть в динамику экспорта.

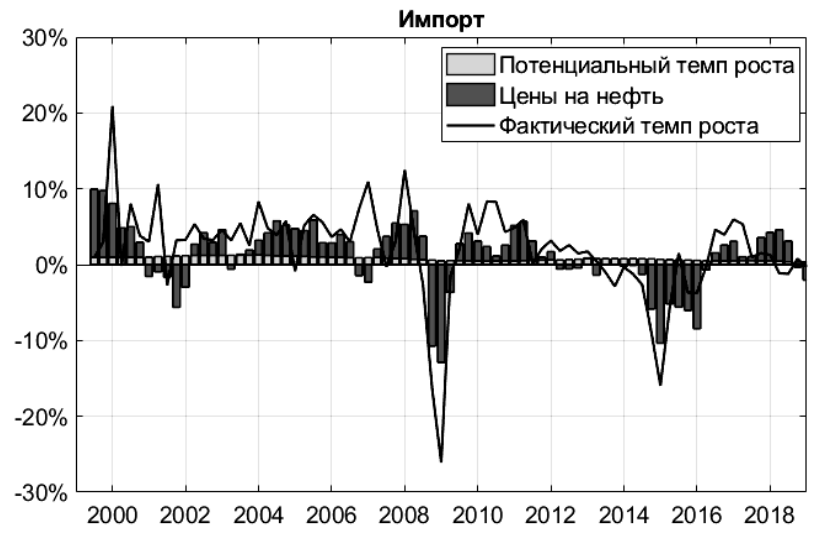

Рис.6. Вклад потенциального темпа роста и цен на нефть в динамику импорта.

\section{5. Заключение}

B работе предложена спецификация VAR модели с дополнительной задачей регуляризации, аналогичной задаче фильтра Ходрика-Прескотта для выделения единого сбалансированного темпа роста структурной компоненты основных российских макроэкономических показателей. Модель позволяет выделить сбалансированный потенциальный темп роста для ВВП без госрасходов, потребления, инвестиций, экспорта и импорта, очищенный от влияния нефтяных цен.

Модель диагностирует замедление потенциального темпа роста российской экономики с 4\% в год в начале 2000 -х до 1.4\% в год в III квартале 2019 г. Оценки модели свидетельствуют о том, что в период 2000-2008 гг. при совокупном росте ВВП без госрасходов на $66 \%$ увеличение цен на нефть кумулятивно дало $17 \%$ роста ВВП без госрасходов, а структурная компонента 35\%. За весь рассмотренный период с 2000 г. по третий квартал 2019 г. цены на нефть и структурная компонента внесли 10 и 61\% в ВВП без госрасходов соответственно, при этом ВВП без госрасходов за весь период вырос на 75\%. 


\section{СПИСОК ЛИТЕРАТУРЫ}

1. Hodrick R.J., Prescott E.C. Postwar US business cycles: an empirical investigation // J. of Money, Credit, and Banking, 1997, v.29, №1, p.1-16.

2. Baxter M., King R.G. Measuring business cycles: approximate band-pass filters for economic time series // Review of economics and statistics, 1999, v.81, №4, p.575-593.

3. Beveridge S., Nelson C.R. A new approach to decomposition of economic time series into permanent and transitory components with particular attention to measurement of the 'business cycle' // Journal of Monetary economics, 1981, т.7, №2, p.151-174.

4. St-Amant P. et al. Measurement of the Output Gap: A discussion of recent research at the Bank of Canada. - Bank of Canada, 1997, №79, 75 p.

5. Синельников-Мурылев С., Дробышевский С., Казакова М. Декомпозиция темпов роста ВВП России в 1999-2014 годах // Экономическая политика, 2014, №5, с.7-37;

Sinel'nikov-Murylev S., Drobyshevskij S., Kazakova M. Dekompoziciya tempov rosta VVP Rossii v 1999-2014 godah // Ekonomicheskaya politika, 2014, №5, s.7-37.

6. Vashchelyuk N., Zubarev A., Trunin P. Determination of the Output Gap for the Russian Economy. - Russ. Presidential Acad. of Nation. Econ. \& Publ. Administration, 2016, 83 p.

7. Орлова Н.В., Егиев С.К. Структурные факторы замедления роста российской экономики // Вопросы экономики, 2015, №12, с.69-84;

Orlova N.V., Egiev S.K. Strukturnye faktory zamedleniya rosta rossijskoj ekonomiki // Voprosy ekonomiki, 2015, №12, s.69-84.

8. Орлова Н.В., Лаврова Н.А. Потенциальный рост как отражение перспектив российской экономики // Вопросы экономики. 2019, № 4, с.5-20;

Orlova N.V., Lavrova N.A. Potencial'nyj rost kak otrazhenie perspektiv rossijskoj ekonomiki // Voprosy ekonomiki, 2019, №4, s.5-20.

9. Villani $M$. Steady-state priors for vector autoregressions // J. of Applied Econometrics, 2009, v.24, №4, p.630-650.

10. Clements M., Hendry D. Forecasting economic time series. - Cambridge Univ. Press, 1998, p.390.

11. King R. et al. Stochastic Trends and Economic Fluctuations // American Economic Review, 1991, v.81, №4, p.819-40.

12. Полбин А.В. Оценка влияния шоков нефтяных цен на российскую экономику в векторной модели коррекции ошибок // Вопросы экономики, 2017, №.10, c.27-49;

Polbin A.V. Ocenka vliyaniya shokov neftyanyh cen na rossijskuyu ekonomiku v vektornoj modeli korrekcii oshibok // Voprosy ekonomiki, 2017, №.10, s.27-49.

13. Пильник Н.П., Радионов С.А., Станкевич И.П. Обобщенная многопродуктовая декомпозиция элементов использования ВВП России // Экономический журнал ВШЭ, 2018, v.22, №2, c.251-274;

Pil'nik N.P., Radionov S.A., Stankevich I.P. Obobshchennaya mnogoproduktovaya dekompoziciya elementov ispol'zovaniya VVP Rossii // Ekonomicheskij zhurnal VSHE, 2018, v.22, №2, c. 251-274.

14. Whelan K. A guide to US chain aggregated NIPA data // Review of income and wealth, 2002, v.48, №2, p.217-233.

15. Полбин А.В., Скроботов А.А. Тестирование наличия изломов в тренде структурной компоненты ВВП РФ // Эконом. журнал ВШЭ, 2016, v.20, №4, c.588-623;

Polbin A.V., Skrobotov A.A. Testirovanie nalichiya izlomov v trende strukturnoj komponenty VVP RF // Ekonom. zhurnal Vysshej shkoly ekonomiki, 2016, v.20, №4, s.588-623.

Поступила в редакцию 15.01.2020

После доработки 17.03.2020

Принята к публикации 20.04.2020 